\title{
Cushing's Syndrome, missed in Pregnancy
}

\author{
K.Jacob' ${ }^{1}$ P.Jinadev ${ }^{2}$ \\ Department of Diabetes \& Endocrinology \\ 'Pilgrim Hospital, United Lincolnshire NHS Trust,Boston,PE 21 9QS \\ ${ }^{2}$ Central Manchester University Hospitals NHS Foundation Trust, M130 JE
}

\section{Case presentation}

- Mrs XY,aged 26years, primigravida was seen in obstetric clinic for mild per vaginal bleed. Ultrasonography confirmed 6 to 8 weeks gestation.

- There was no obvious cause for vaginal bleed. The blood test revealed polycythaemia which was transient.

- She had $75 \mathrm{gm}$ oral glucose tolerance test at 17 weeks and 28weeks gestation.

- The test at 28 weeks was borderline positive for gestational diabetes.

$75 \mathrm{gm} \mathrm{OGTT}$ at 28 weeks gestation Glucose $\mathrm{mmol} / \mathrm{l}$ 08:49 am 4.9

$11 \mathrm{am}$ 8.4

- She was referred to the diabetes specialist nurse. She given dietary advice and was taught self monitoring of capillary blood glucose.

- HbA1c was $31 \mathrm{mmol} / \mathrm{mol}$ but home glucose monitoring remains high. She was commenced on Metformin.

- At 31 weeks, she developed oral thrush. She required topical Nystatin.

- Blood pressure was elevated at 148/97 mmHg.Labetalol was commenced.

- At 35 weeks, blood pressure remains high at 154/99mmHg. She was already on Nifidepine $10 \mathrm{mg}$ bd and Labetalol 200mg qid. Urine showed proteinuria. A diagnosis of pre-eclampsia was made.

- At 36 weeks she underwent an emergency lower segment caesarean section.

- Six months postpartum she was referred to Endocrinology clinic for persisting hypertension and facial swelling.

- On examination, she had proximal myopathy, easy bruising and purplish abdominal striae.

- Blood pressure was controlled on Labetalol.

- BMI 21.2, HbA1c $31 \mathrm{mmol} / \mathrm{mol}$.

\section{Investigations}

- 24 hr urinary catecholamines were normal.

- Androgen profile was normal.

- Comparison of facial and body features with photographs taken pre-pregnancy was quite striking

\section{4 hour urine free cortisol}

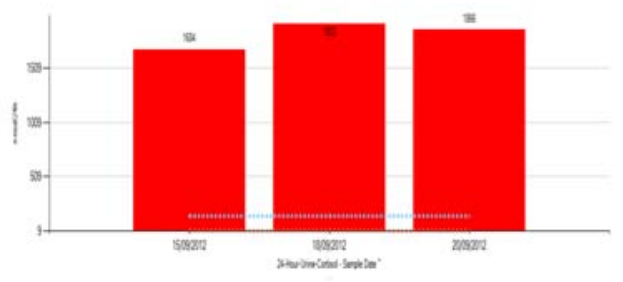

- $1 \mathrm{mg}$ overnight Dexamethasone suppression test was unsuppressed at $580 \mathrm{nmol} / \mathrm{l}$.

- Low dose Dexamethasone suppression test didn't show suppression.

- 9am ACTH levels were suppressed.

- CT scans confirmed right adrenal adenoma.

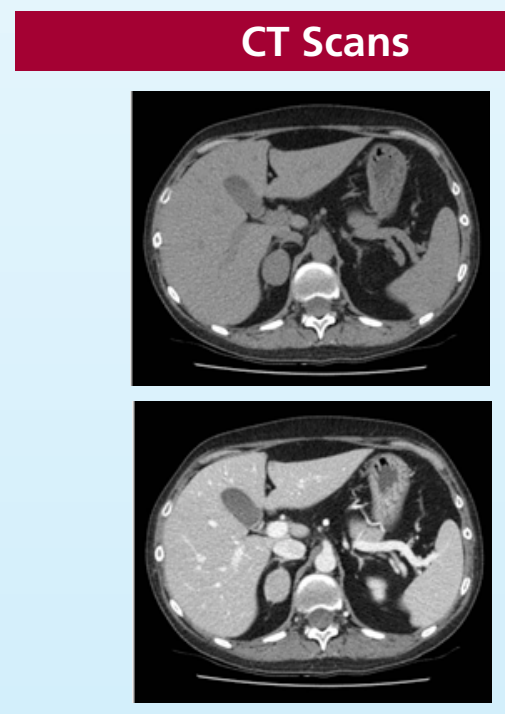

- A diagnosis of ACTH independent Cushing's syndrome was made.

$\quad$ Management
Ketoconazole was commenced for
inhibition of steroidogenesis.
She underwent laparoscopic right
adrenalectomy.
Histology confirmed adrenocortical
adenoma.

\section{Outcome and follow up}

- Post surgery her blood pressure normalised.

- The facial as well as body features are much improved.

\section{Discussion}

- The signs and symptoms of hypercortisolemia overlap with normal pregnancy making it difficult to diagnose.

- Pregnancy itself is a state of relative hypercortisolemia.

- The serum total and free cortisol as well as $24 \mathrm{hr}$ urinary free cortisol excretion is increased in pregnancy. Plasma ACTH are increased due to increased placenta secretion of $\mathrm{CRH}$.

- The hypercortisolemia of Cushing's syndrome interferes with menstrual cycle and very rarely results in pregnancy. If pregnancy occurs in Cushing's syndrome, it can result in significant maternal and foetal morbidity as in our case.

- ACTH independent Cushing's syndrome is more common in pregnancy.

- It is quite possible the 'pure cortisol' secreting adenomas interfere less with reproductive function than the ' mixed cortisol \& androgen excess' as would be seen with Cushing's disease or adrenal carcinoma.

- The foetus is partially protected from maternal hypercortisolemia by 11 beta hydroxysteroid dehydrogenase.

\section{Conclusion}

- Majority (60\%) of Cushing's in pregnancy is ACTH independent( $45 \%$ adenoma \& $15 \%$ carcinoma) (1-4)

- A high index of suspicion is necessary to consider Cushing's syndrome in pregnancy especially if there is excessive weight gain, along with combination of gestational diabetes and hypertension as in our patient

\section{References}

Aron DC, Schnall AM, Sheeler LR. Cushing's syndrome and pregnancy. Am J Obstet Gynecol 1990; 162:244. Buescher MA, McClamrock HD, Adashi EY. Cushing syndrome in pregnancy. Obstet Gynecol 1992; 79:130.

Buescher, MA. Cushing's syndrome in pregnancy. Endocrinologist 1996; 6:357.

Lindsay JR, Jonklaas J, Oldfield EH, Nieman LK. Cushing's syndrome during pregnancy: personal experience and review of the literature. J Clin Endocrinol Metab 2005: 90:3077. 